\title{
Evidence based for exercise program in physical therapy in patients with multiple sclerosis
}

\section{INTRODUTION:}

Multiple Sclerosis is a disease of the central nervous system that affects more frequently young women. It is a progressive and unpredictable disease, resulting in some cases of disabilities and limitations to physical, psychological and social level.

\section{OBJECTIVES:}

To review the literature for evidence based of the effectiveness of physiotherapy intervention in Multiple Sclerosis.

\section{METHODS:}

The research was performed in the databases SCIELO, MEDLINE and PEDRO, where articles in English and Portuguese idioms published from 2003 to 2013 were selected. We used the following keywords: "Multiple Sclerosis, Exercise and Physiotherapy", having as result 58 articles. We applied the following Exclusion criteria in the selection of articles: All artcles that did not register data recommendation for exercise, all exercise programs that are applied to patients with EDSS less than 7 and those patients submitted to pharmacological tests. At the end articles 26 were selected.
Pio Gervásio $1-2$ / Luísa Pedro 1 , Escola Superior de Tecnologia da Saúde de Lisboa -Portugal ${ }_{2}$ Força Aérea Nacional de Angola - Luanda - Angola

\section{RESULTS:}

Exercise programs in physical therapy for a successful management of functional limitations, disabilities, and to improve the quality of life of patients with multiple sclerosis. Various studies of exercise in multiple sclerosis recommend session, alternating with rest periods, two to three times per week. For people with EDSS index less than 7. Several studies have implemented specific programs to months. The number of series of exercises should start with 1-3 series, progressively increasing to 3-4 series. The resting between series of exercises should be 2-4 minutes. Some authors advise the combination of aerobic and strength training with 3 sessions / week of strength training and 1 session of aerobic exercise.

\section{CONCLUSIONS:}

Through the reading of results we can see that exercise programs in physical therapy plays a key role in the prevention and rehabilitation of individuals with multiple sclerosis.

\section{IMPLICATIONS:}

The basis of scientific evidence of exercise for the intervention of physiotherapy treatments in patients with multiple sclerosis programs is critical to the effectiveness of treatments. 